\title{
Quantitative Evaluation of Surface Crack Depth with Laser Spot
}

\author{
Thermography \\ Jinxing Qiu, Cuixiang Pei*, Haochen Liu and Zhenmao Chen ${ }^{*}$ \\ State Key Laboratory for Strength and Vibration of Mechanical Structures, \\ Shaanxi Engineering Research Center of NDT and Structural Integrity Evaluation, \\ Xi'an Jiaotong University, Xi'an, China
}

\begin{abstract}
In this study, a numerical method based on finite element method (FEM) is developed to simulate the heat flow generated by laser spot source and investigate the relationship between crack size and temperature distribution. The feasibility of the simulation method is validated by experiments both in time and spatial domains. The simulation and experiment results also show that the crack depth can be described by two characteristic parameters. Furthermore, a quantitative retrieval method based on neural network is developed for the crack depth evaluation by using the parameters. By using the proposed method, crack depth can be determined only by analyzing measured surface temperature values. Keywords: Laser spot thermography; Surface crack; Quantitative evaluation; Neural network
\end{abstract}

\section{Introduction}

Detection of surface breaking cracks is an important task to ensure the safe operation of the key structures like nuclear plants. Besides the widely used NDT techniques, such as ultrasonic testing (UT) and eddy current testing (ECT), laser spot thermography (LST) is a novel remote inspection

\footnotetext{
${ }^{*}$ Corresponding authors.

Email address: pei.cx@mail.xjtu.edu.cn (C. Pei); chenzm@mail.xjtu.edu.cn (Z. Chen).
} 
method for surface crack testing and imaging [1-2]. Comparing with conventional infrared thermography NDT method which uses flash or halogen lamps to heat, LST has the advantages of being deployable remotely, adjusting the focus position explicitly, and being suitable for the detection of surface crack as the heat flow mainly propagates near the surface.

Some researchers have investigated the imaging method by simulation and experiments. In recent work by Teng Li et al. [3], a crack imaging technique is developed based on second derivative image processing of thermal images. Y. An et al. proposed a crack imaging and extraction process using the holder exponent analyses [4]. From processed thermal images, the crack position and length can be determined. Moreover, some approaches to sizing cracks by pulse thermography or lock-in thermography have also been introduced. J. Schlichting et al. exploit the asymmetry of the thermal footprint of a laser spot affected by the crack [5]. M. Streza et al. use the saturation level of Laplacian of the amplitude image to estimate crack depth [6]. However, each of them has some disadvantages, such as the frequent use of calibration specimens and low quantitation accuracy.

In this paper, a numerical method based on finite element method (FEM) is applied to simulate the laser induced heat flow and investigate the relationship between the crack size and temperature distribution, which is validated by experiments. Two characteristic parameters that highly depend on crack depth are extracted from surface temperature values near the laser heated region to evaluate the crack depth. A quantitative estimation method based on neural network is finally developed to evaluate the

crack depth. By applying temperature data from simulation to the neural network model, the feasibility of this method is validated.

\section{Simulation method and experimental validation}

\subsection{Simulation method}

To investigate the relationship between crack dimension and surface temperature field, a numerical 
simulation method and an original code based on FEM is developed. In this work, the simulation model is considered as a cuboid specimen. The surface of specimen is heated by laser spot source and a transient, non-uniform temperature field is induced. For homogeneous isotropic materials, the 3D thermal conduction equation can be described as:

$$
\rho c \dot{\boldsymbol{T}}-\kappa \nabla^{2} \boldsymbol{T}=0
$$

where $\rho$ is the density $\left(\mathrm{kg} / \mathrm{m}^{3}\right), c$ the thermal capacity $(\mathrm{J} / \mathrm{kg} \cdot \mathrm{K}), \kappa$ the thermal conductive coefficient $(\mathrm{W} / \mathrm{m} \cdot \mathrm{K})$, and $T$ the temperature $(\mathrm{K})$.

The boundary condition of the temperature field can be expressed as:

$$
n_{x} \cdot \frac{\partial T}{\partial x}+n_{y} \cdot \frac{\partial T}{\partial y}+n_{z} \cdot \frac{\partial T}{\partial z}=-\frac{q}{\kappa}
$$

where $n_{i}$ is the component of normal vector in $i$ th direction and $q$ the laser heat flux source $\left(\mathrm{W} / \mathrm{m}^{2}\right)$. According to FEM, the governing equation can be established and the equation can be solved with the integration method in time domain [7].

\subsection{FEM simulation results and experimental validation}

Fig. 1 shows the mesh for a cuboid specimen model in simulations. The model is uniformly meshed in $\mathrm{x}$ and $\mathrm{y}$ direction while in $\mathrm{z}$ direction, only the top surface is finely meshed. A total of 880,000 elements and 929,223 nodes are generated to ensure the accuracy. Material of specimens used in the simulation and experiment is 304L stainless steel and the crack area is regarded as air. By setting the material type of specific elements as air, the different location and size of cracks can be simulated. Main thermodynamic parameters used in simulations can be found in Table 1. Fig. 2 shows the simulated heat flow induced by a laser spot source at point $\mathrm{O}$ in the model without crack and with crack. The two models have the same dimensions (a cuboid in $20 \mathrm{~mm} \times 20 \mathrm{~mm} \times 10 \mathrm{~mm}$ ) and material (304L stainless steel). The difference is that in Fig. 2 (b) there is an open rectangle crack on 
the surface. The crack located at $1.6 \mathrm{~mm}$ to the center and has a length of $10 \mathrm{~mm}$, opening of $0.4 \mathrm{~mm}$ and depth of $3 \mathrm{~mm}$.

Experimental validation for the FEM simulation was conducted both in time and spatial domains. Fig. 3 shows the experimental setup. The experimental system mainly contains a high energy pulse laser, a signal generator to modulate laser pulse shape and duration and an IR camera with resolution of $0.05 \mathrm{~K}$ to capture thermal graphs. The laser pulse width in simulation and experiment is $4 \mathrm{~s}$, and the radius of the laser spot is $0.5 \mathrm{~mm}$. The peak power of laser spot is $20 \mathrm{~W}$. Temperature values at the top surface are recorded by the IR camera during the heating time.

Table 1. Material properties used in simulation.

\begin{tabular}{c|c|c}
\hline Material & 304L Stainless steel & Air \\
\hline Thermal conductivity $(\mathrm{W} / \mathrm{m} \cdot \mathrm{K})$ & 13.5 & 0.025 \\
\hline Specific heat $(\mathrm{J} / \mathrm{kg} \cdot \mathrm{K})$ & 485 & 1005 \\
\hline Density $\left(\mathrm{kg} / \mathrm{m}^{3}\right)$ & 7900 & 1.205 \\
\hline
\end{tabular}

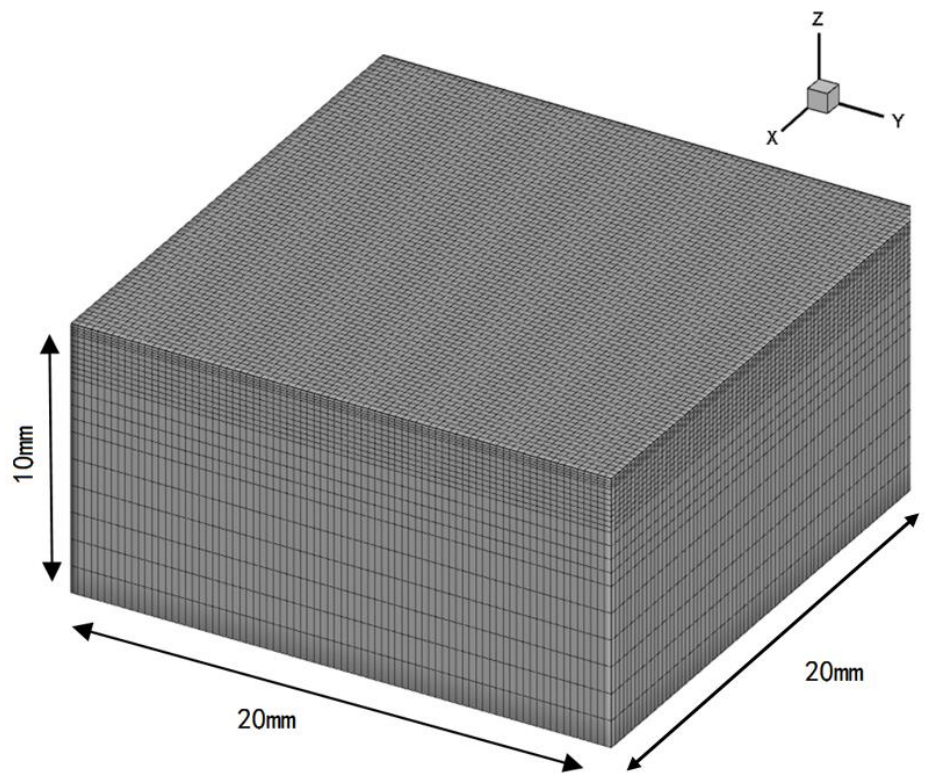

Fig. 1. Mesh for simulations. A finer mesh is used at the top surface as the heat flow mainly propagates near the surface. 

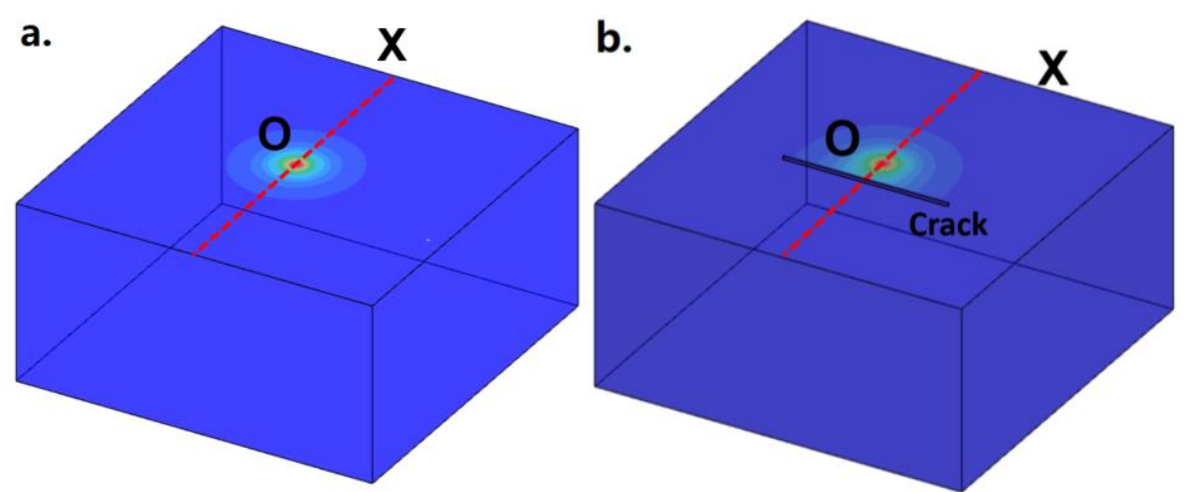

Fig. 2. 3D Simulation of the heat flow induced by a laser spot source in the model (a) without crack and (b) with crack

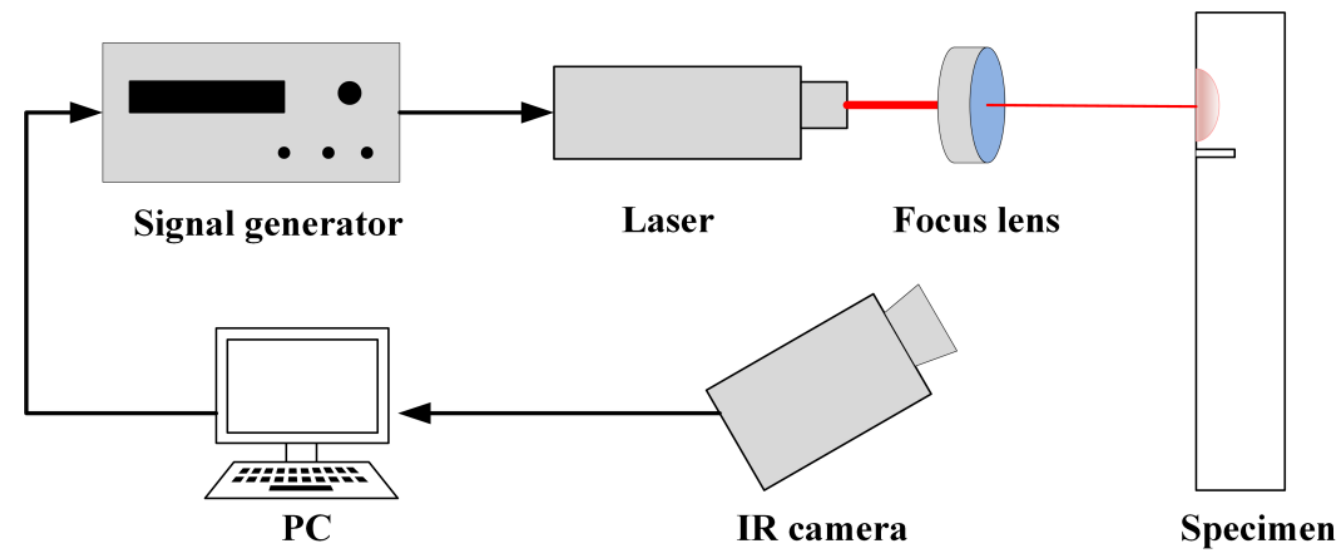

Fig. 3. Experimental setup for the LST system

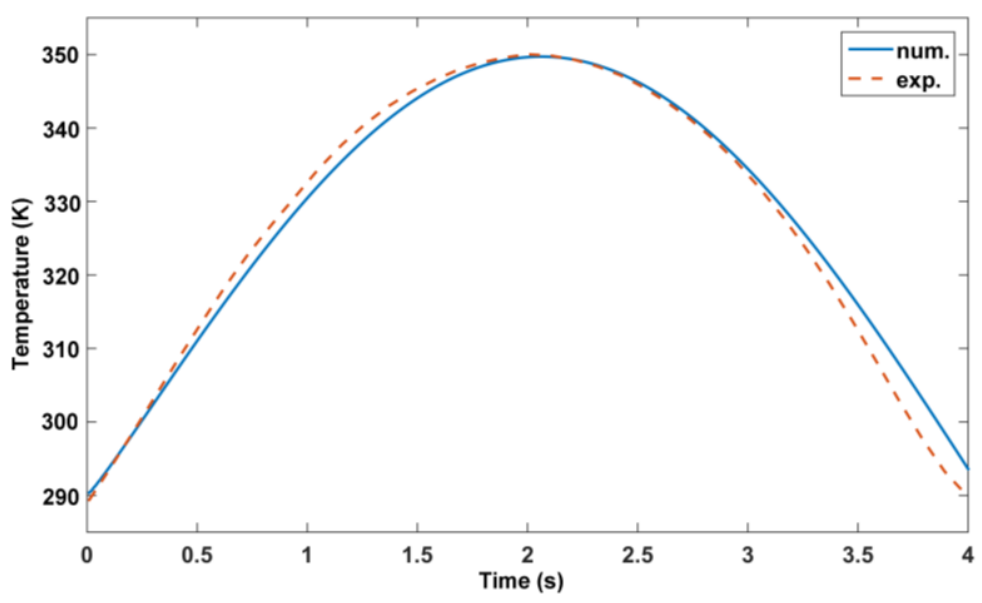

Fig. 4. Temperature values at point $\mathrm{O}$ of Fig. 2 (a) in simulation and experiment during the heating time. The specimen surface is excited by a $4 \mathrm{~s}$ sinusoidal wave pulse. 

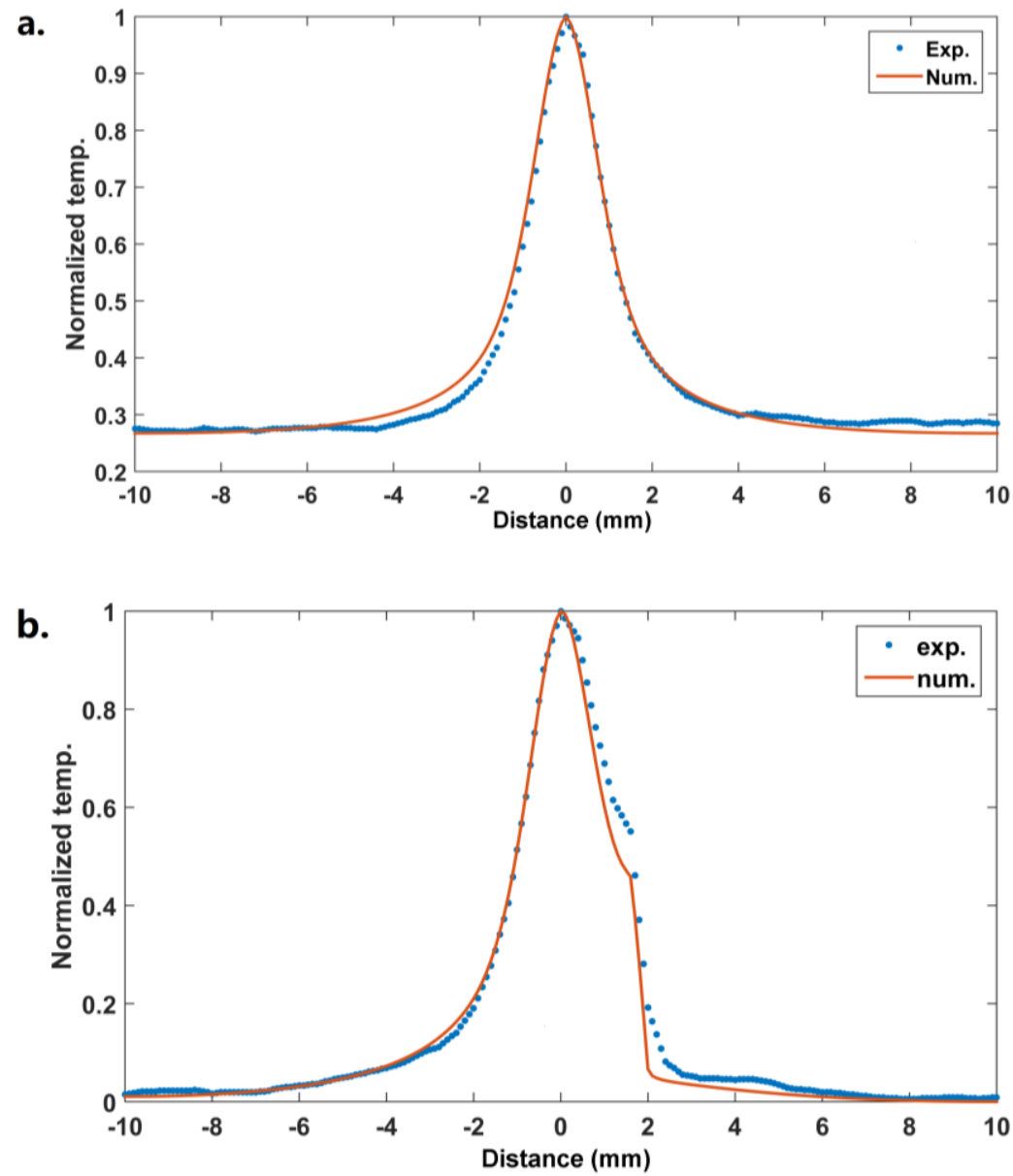

Fig. 5. Comparisons of temperature profile in simulation and experiment

Fig. 4 compares the simulation and experiment results in time domain. Temperature changes at laser spot center agree well in simulation and experiment during the heating time when excited by sinusoidal wave pulse.

Fig. 5 (a) compares the temperature profile on line $X$ in simulation and experiment. In Fig. 5 (b), simulation and experiment temperature profiles for a crack model are also compared. The simulation and experiment condition is the same with that for a no crack model but using a specimen with a surface crack (see Fig. 2 (b)). The crack located $1.6 \mathrm{~mm}$ to the spot center on top surface has a length of $10 \mathrm{~mm}$, width $0.4 \mathrm{~mm}$ and depth $3 \mathrm{~mm}$. The measurement result shows a good approximation to the simulation result, which adds to the validation of the simulation codes and provides an approach to investigating the crack-caused asymmetries in temperature distribution for different crack sizes and 
specimen models.

\section{Quantitative evaluation method}

\subsection{Parameters for depth evaluation}

The distribution of surface temperature field mainly depends upon crack dimension (length, opening, and depth) and distance from laser spot to crack. By a crack imaging and extraction process, the crack length, width and position can be determined, which means that in the depth evaluation process, the crack length, width and position are constant. In this precondition simulation and analysis were conducted and the results suggest that some parameter values can be extracted from surface temperature to estimate the crack depth.

Fig. 6 (a) shows the simulated thermal images of no crack region and near crack region by FEM. A1, A2 are the line profiles taken through the center of the thermal image. As shown in Fig. 6 (b), in the left part A1 is almost the same with A2, while in the right part obvious differences can be found. The difference of temperature distribution can be described by 'area' difference $\mathrm{S}$ and calculated by equation (3).

$$
S=\sum_{i=0}^{10 / \Delta i}\left|0.5 \cdot\left(A 1_{i}+A 1_{i+1}\right) \cdot \Delta i-0.5 \cdot\left(A 2_{i}+A 2_{i+1}\right) \cdot \Delta i\right|
$$

where $A 1_{i}$ and $A 2_{i}$ denote the $i$ th temperature value on line $A 1$ and $A 2$ respectively. $\Delta i$ is the distance between two adjacent measurement points. In this equation the crack-caused distinction of temperature distribution is described as 'area' difference.

Another characteristic parameter $\Delta \mathrm{T}$ is the difference of mean temperature value in region $\mathrm{B} 1$ and B2 (see Fig. 7) that are symmetric around laser spot:

$$
\Delta \boldsymbol{T}=\boldsymbol{T}_{\text {average }}^{B 1}-\boldsymbol{T}_{\text {average }}^{B 2}
$$

where $T_{\text {average }}$ denotes the mean temperature value in a region. $\mathrm{B} 1$ and $\mathrm{B} 2$ are rectangle regions that 
have a distance $m=2.1 \mathrm{~mm}$ to the spot center and have the size $A=0.5 \mathrm{~mm} \times 10 \mathrm{~mm}$. Fig. 8 shows typical simulation results of the specimen model with cracks in different depths $d=0.5 \mathrm{~mm}, 1.0 \mathrm{~mm}, 2.0$ $\mathrm{mm}$, and $3.0 \mathrm{~mm}$. Parameter $\Delta \mathrm{T}$ and $\mathrm{S}$ are respectively normalized by its maximum value when $d=$ $3.0 \mathrm{~mm}$. The results show a monotone relationship between depth and the parameters during the heating period.

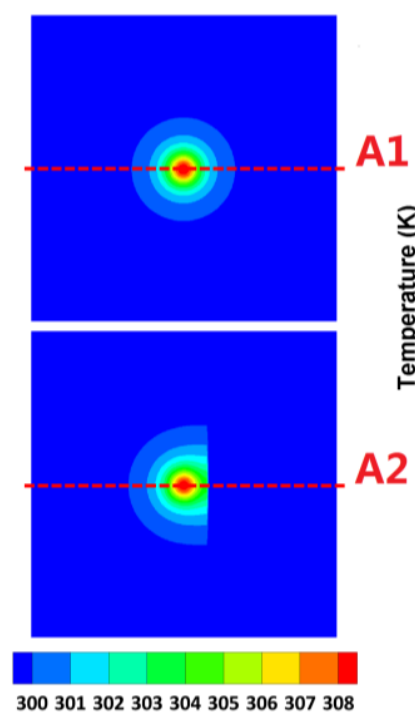

a

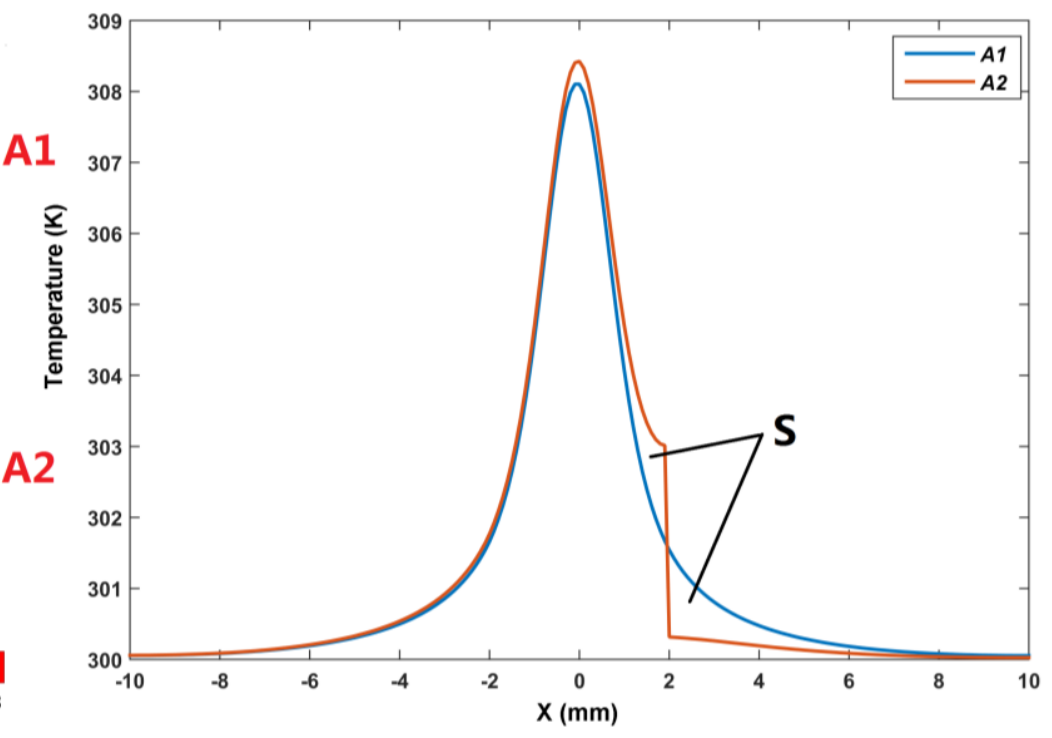

b

Fig. 6. (a) Simulated thermal images of no crack region and crack region. (b) The temperature profile across the crack and the center of the spot.

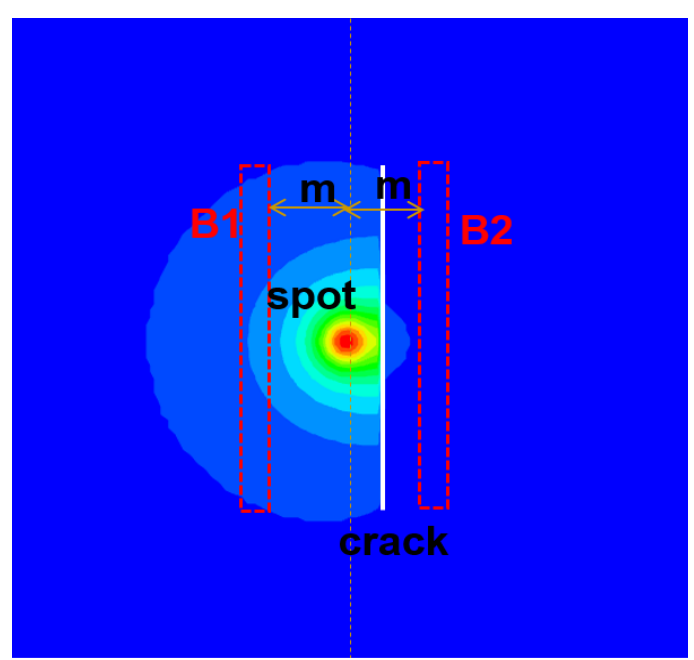

Fig. 7. Reference region B1 and B2 that are symmetric around laser spot. 
a.

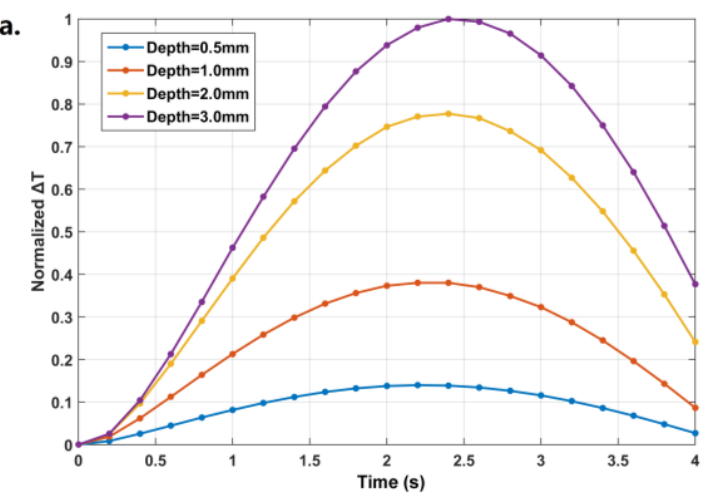

b.

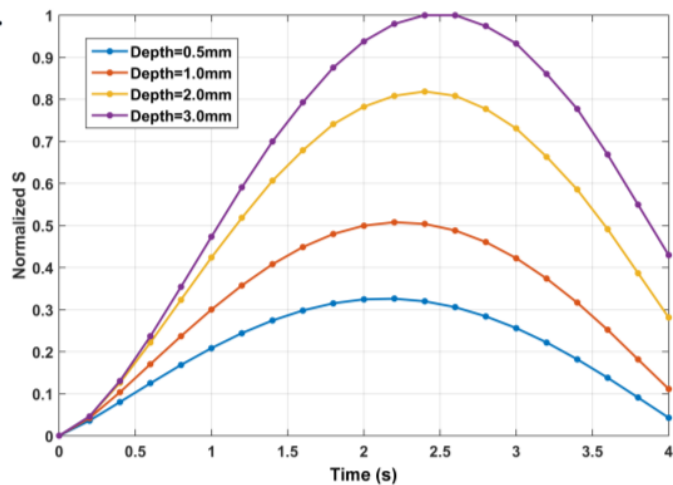

Fig. 8. Simulation results of relationship between crack depth and (a) $\Delta \mathrm{T}$ and (b) $\mathrm{S}$ at different times

a.

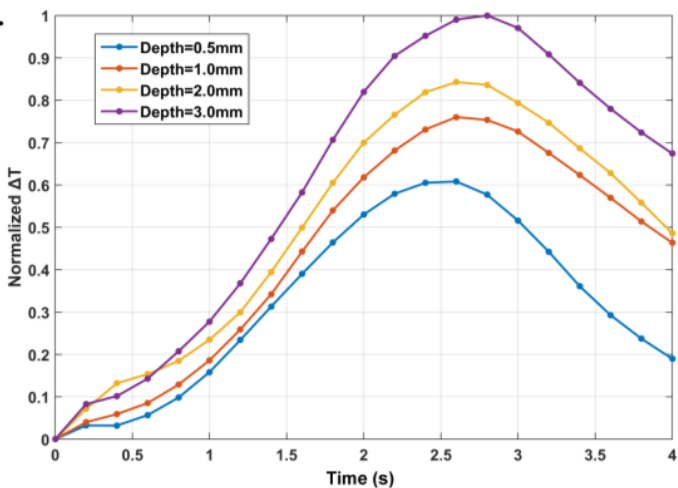

b.

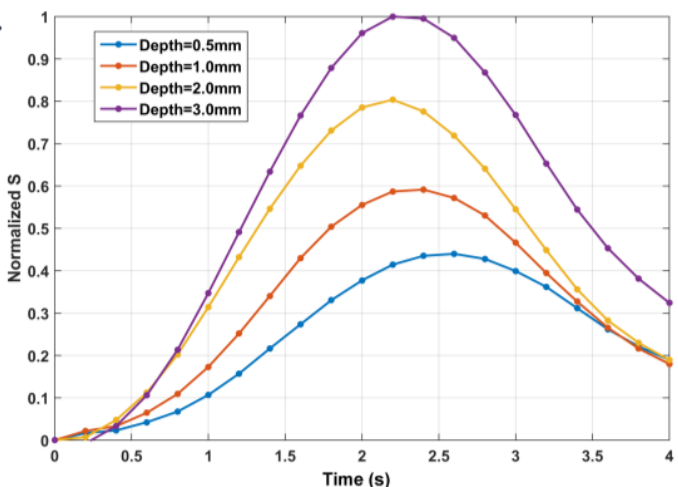

Fig. 9. Experiment results of relationship between crack depth and (a) $\Delta \mathrm{T}$ and (b) $\mathrm{S}$ at different times

To verify the suitability and calibrate the data analysis procedure, we used a stainless steel test specimen. The specimen has a size of $100 \times 100 \times 20 \mathrm{~mm}^{3}$ and is produced with four spark eroded notches with the width $w=0.4 \mathrm{~mm}$, length $l=20 \mathrm{~mm}$ and depth $d=0.5 \mathrm{~mm}, 1.0 \mathrm{~mm}, 2.0 \mathrm{~mm}$, and 3.0mm respectively. Fig. 9 shows the parameters at different times obtained by experiment using the specimen. The parameter values are normalized by the maximum value when $d=3.0 \mathrm{~mm}$. The curves measured in experiments do not accurately agree with that in simulation because of the sensitivity of the IR camera and the uneven surface pattern of specimen. However, similar trends for the behavior of characteristic parameters are exhibited for experimental measurements and FEM simulations.

\subsection{Crack depth evaluation algorithm based on neural network}

To establish the mapping relationship between depth and parameters, the conventional method is to 
compare the parameter-depth curve to a calibration curve, which has to be determined by experiment using a set of test specimens. Any parameter of the crack dimension changes, the calibration curve should be determined once again.

A neural network (NN) model is trained by using the above two characteristic parameters extracted from simulated data. In this section we briefly use the fundamentals of $\mathrm{NN}$ as a mathematic tool to obtain the solution from the multi-to-one mapping relation and nonlinear functions [8-9]. The network used in this work was a 6-5-1 feedforward neural network (see Fig. 10) and achieved by neural network package provided by MATLAB. The input values of neural network are characteristic parameter values at different times and the output value is crack depth. Input values at $t=0.5 T_{\text {pulse }}\left(T_{\text {pulse }}\right.$ denotes pulse width), $t=T_{\text {pulse }}$ and $t=1.5 \mathrm{~T}_{\text {pulse }}$ are sufficient to train the network and the accuracy will not be significantly improved by increasing the number of input values.

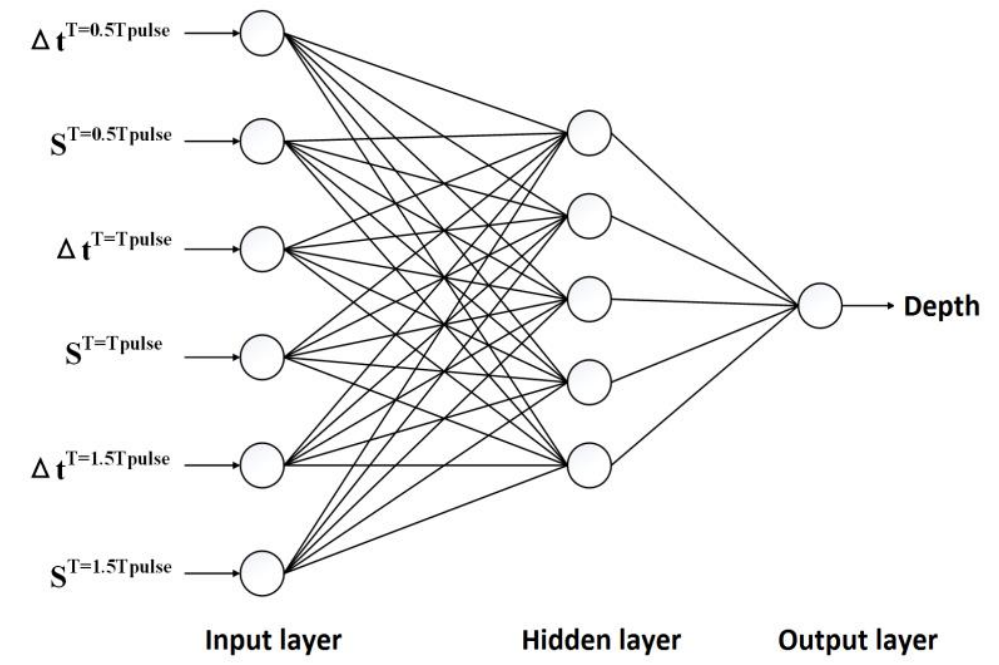

Fig. 10. Topological structure of the neural network used for depth evaluation

\subsection{Results and discussion}

Simulated temperature values of specimen with cracks with opening $0.4 \mathrm{~mm}$, length $10 \mathrm{~mm}$ and depth ranging from $0.2 \mathrm{~mm}$ to $3.0 \mathrm{~mm}$ in $0.2 \mathrm{~mm}$ step are used to train the network. Data with crack depth in $0.5 \mathrm{~mm}, 1.0 \mathrm{~mm}, 1.5 \mathrm{~mm}, 2.0 \mathrm{~mm}, 2.5 \mathrm{~mm}$ and $3.0 \mathrm{~mm}$ are used as testing data to assess the 
accuracy of network. The depth evaluation results can be found in Table 2, which is very promising.

Table 2. Estimated depth by the simulation.

\begin{tabular}{c|c|c}
\hline True depth (mm) & Estimated depth $(\mathrm{mm})$ & Relative error \\
\hline 0.5 & 0.4793 & $-4.14 \%$ \\
\hline 1.0 & 1.0336 & $3.36 \%$ \\
\hline 1.5 & 1.4514 & $-3.24 \%$ \\
\hline 2.0 & 2.0313 & $1.57 \%$ \\
\hline 2.5 & 2.5727 & $2.91 \%$ \\
\hline 3.0 & 2.8800 & $-4.00 \%$ \\
\hline
\end{tabular}

\section{Conclusions}

In this paper, a simulation method based on FEM is presented to simulate the temperature distribution in laser spot thermography and its feasibility is validated by experiments. Characteristic parameters $\Delta \mathrm{T}$ and $\mathrm{S}$ that have intimate correlation with crack depth are also introduced. Numerical simulation and experimental measurement results show that the two parameters can be used as indicators of the crack depth. Moreover, a data analysis method based on neural network is also developed to determine crack depth by using the parameters. In this method, the crack depth can be determined only by measuring the surface temperature and analysis the characteristic parameter values. A simulation experiment was also conducted to evaluate the depth of different cracks. The relative error of evaluation results are all in 5\%, which demonstrates the validity of the proposed method.

The investigation of depth evaluation by using experimental measured characteristic parameters has not been conducted because of the errors caused by the low sensitivity of the IR camera and the uneven surface pattern of specimens. Thus, our further work will focus on improving detection precision. 


\section{Acknowledgment}

The authors would like to thank the National Magnetic Confinement Fusion Program of China (No. 2013GB113005), National Natural Science Foundation (No. 11502192, 51277139, 51577139), National Basic Research Programs of China (No. 2011CB610303) and Postdoctoral Science Foundation Program of China (No. 2015M570819) for funding.

\section{References}

[1] Maldague X. P. and Marinetti S., Pulse Phase Infrared Thermography, J. Appl. Phys., 79 (1996), 2694-2698.

[2] B. Weekes, P. Cawley, D. P. Almond, and T. Li, The effect of crack opening on thermosonics and laser spot thermography, AIP Conference Proceedings, 1211490 (2010).

[3] Teng Li, Darryl P. Almond, D. Andrew S. Rees, Crack imaging by scanning pulsed laser spot thermography, NDT\&E International 44 (2011) 216-225.

[4] Yun-Kyu An, Ji Min Kim, Hoon Sohn, Laser lock-in thermography for detection of surface-breaking fatigue cracks on uncoated steel structures, NDT\&E International 65 (2014) 54-63.

[5] J. Schlichting, Ch. Maierhofer, M. Kreutzbruck, Crack sizing by laser excited thermography, NDT\&E International 45 (2012) 133-140.

[6] M. Streza, D. Dadarlat, et al., Depth estimation of surface cracks on metallic components by means of lock-in thermography, Rev. Sci. Instrum. 84, 074902 (2013).

[7] Cuixiang Pei, et al., Simulation of surface cracks measurement in first walls by laser spot array thermography, Fusion Engineering and Design (2015), Volumes 109-111, Part B, 1 November 2016, Pages 1237-1241.

[8] Xavier MaIdague, Yves Largoutit, Jean-Pierre Couturier, A study of defect depth using neural networks in pulsed phase thermography: modelling, noise, experiments, Rev. Gen. Therm. 37 (1998) 704-717.

[9] M. B. Saintey, D. P. Almond, An artificial neural network interpreter for transient thermography image data, $N D T \& E$ International 30 (1997) 291-295. 
2019-02-28

\section{Quantitative evaluation of surface crack depth with laser spot thermography}

Qiu, Jinxing

Elsevier

Qiu J, Pei C, Liu H, Chen Z. (2017) Quantitative evaluation of surface crack depth with laser spot thermography. International Journal of Fatigue, Volume 101, Issue 1, August 2017, pp. 80-85 https://doi.org/10.1016/j.ijfatigue.2017.02.027

Downloaded from Cranfield Library Services E-Repository 